\title{
Feasibility of balloon sinuplasty in patients with chronic rhinosinusitis: the Graz experience*
}

\author{
Peter Valentin Tomazic ${ }^{1}$, Heinz Stammberger ${ }^{1}$, Hannes Braun', \\ Walter Habermann' ${ }^{1}$ Christoph Schmid², Georg Philipp Hammer', \\ Wolfgang Koele ${ }^{1}$
}

Rhinology 51: 120-127, 2013 DOl:10.4193/Rhino12.194

*Received for publication: November 12, 2012

Accepted: January 20, 2013

\section{Summary}

Background: Balloon sinuplasty (BSP) is a catheter-based technique to dilate sinus ostia and drainage pathways to create ventilation and drainage. The aim of this study was to evaluate the feasibility of BSP in routine treatment of patients suffering from chronic rhinosinusitis (CRS).

Methodology: Patients with CRS refractory to medical therapy who had been scheduled for endoscopic sinus surgery between 2009 and 2011 were included in this study.

Results: Forty-five consecutive patients were included in this study, in whom 112 sinuses were approached by BSP. Of the 112 sinuses, 68 (60\%) were planned as a "Balloon-Only" procedure and 44 (40\%) were planned as a "Hybrid" procedure. Of the 68 sinuses in the "Balloon-Only" group, in 44 sinuses BSP failed, equating to a failure rate of $65 \%$. Forty-four sinuses were planned for "Hybrid" procedures. In 29 of these sinuses BSP failed, giving a failure rate of $66 \%$.

Conclusion: According to literature, BSP can be a useful adjunct technique to standard FESS. In our experience, however, a failure rate of $65 \%$ for "Balloon-Only" and of $66 \%$ for "Hybrid" procedures occurred, which was regarded as unacceptable by the study group. Therefore, the study initially scheduled for 200 consecutive patients, was abandoned.

Key words: balloon sinuplasty, chronic rhinosinusitis, feasibility, success rate, failure rate

\section{Introduction}

Since its introduction, balloon sinuplasty (BSP) has remained a controversial topic among rhinologists and sinus surgeons around the world, due to a strong media profile and marketing strategies ${ }^{(1,2)}$ followed by some highly enthusiastic reports and promising results in the literature ${ }^{(3-5)}$.

The concept of balloon catheterization has long been used in urology and cardiology. In ENT, Fogarty catheters were used for sinus dilatation some years before Acclarent (Acclarent Inc., Menlo Park, CA, USA) presented their system ${ }^{(1)}$. In this technique, a balloon is advanced over a guide catheter and inflated when the correct position of the catheter is confirmed by either fluoroscopy or, more recently, transillumination. Through the pressure applied, the main respective sinus cavity's ostium is dilated, the thin bone fragments of the smaller adjacent sinus cells are out-fractured and hopefully remodeled so that both 
function and mucosa are preserved. Thus ventilation and drainage pathways of the sinus are created, a principle also followed by classical functional endoscopic sinus surgery (FESS), in which in contrast to BSP, diseased tissue is also resected.

This study was designed to analyse the feasibility of BSP for routine use in patients suffering from chronic rhinosinusitis who otherwise would have been treated with standard FESS procedures.

\section{Materials and methods}

\section{Study population}

The study was approved by the Ethics Committee of the Medical University Graz (Approval Number: 23-482ex10/11).

Initially 200 consecutive patients were scheduled to be included in this study of Balloon sinuplasty (BSP) at the ENT Department of the University Hospital Graz starting 1st January 2009. Patients had to be over 18 years old and free from other chronic or systemic disease. Pregnancy or risk of pregnancy was excluded in female patients. All patients suffered from chronic rhinosinusitis without polyposis (CRSsNP) as defined in the EPOS guidelines ${ }^{(6)}$. A pre-trial study with 37 patients was performed not to bias the current study by a learning curve. All surgeons participating in this study were experienced endoscopic sinus surgeons. Considering the literature ${ }^{(7-9)}$ and our pre-trial results, patients with diffuse eosinophil-mediated chronic rhinosinusitis (eCRS) with polyps were not included since BSP is not suitable for this entity and standard instruments are required in any case.

Patients were referred to our department for surgery by other ENT specialists after failure of conservative medical treatment, which for the individual case usually included topical- and/ or systemic steroids and/or antibiotics. After evaluation of the patients' paranasal sinus CT scans reconstructed in three planes (sagittal, axial and coronal) and clinical examination, our surgeons had to decide pre-operatively whether BSP for the frontal, maxillary and sphenoid sinuses was considered suitable in the respective case (absence of: unusual anatomy, osteomas, fungus balls, mucoeceles, massive osteoneogenesis, suspicion of malignant or other benign tumours) and which approach ("Balloon-Only" or Hybrid") had to be performed on a single or a combination of more sinuses, depending on the extent of disease. "Balloon-Only" meant that only the balloon system was used to perform the operation. "Hybrid" meant that in addition to the balloon equipment, standard instruments were used for initial steps such as uncinectomies, or bullectomies. If required, the remaining anterior ethmoid and posterior ethmoid sinuses were approached with standard FESS instruments after BSP. “Hybrid” procedures were chosen if middle meatus anatomy was narrow and one would not be able to advance guide catheters into the middle meatus and/or ethmoidal infundibulum. For "Hybrid" procedures in the sphenoid, the turbinates were gently deflected laterally with a Freer's elevator if one could not advance catheters towards the natural sphenoid sinus ostium if the access was narrow in the common nasal meatus.

\section{Intervention}

All BSPs were performed as recommended by the company (Acclarent Inc.) and described elsewhere ${ }^{(7,10)}$. All surgeons were very experienced sinus surgeons. Two surgeons (HS and $\mathrm{HB}$ ) were trained and certified by the company (www.balloonsinuplasty. com/find-an-ent-doctor/international-physicians/\#Austria) and instructed the remaining two surgeons (WK and WH). All equipment was provided by Acclarent (Acclarent Inc.). In a pre-study trial, the technique was practiced so as not to bias the data by a learning curve. All interventions were supervised by the study coordinator (PVT). All BSP interventions were performed with the Relieva Luma system for transillumination, no C-arm fluoroscopy was used. At least four cannulation attempts were done for each sinus and when successful, the balloon was inflated with sodium chloride $0.9 \%$ up to a maximum of 12 atmospheres and was kept inflated for 10-30 seconds. All patients signed informed consent prior to surgery. Following data were analysed: gender, age, CT-score according to Lund-Mackay ${ }^{(11)}$, previous surgeries (yes or no, which approach: endoscopic/external or both), intra- and postoperative adverse events, success and failure rates. Bone thickness was analysed on CT-scans and was referred to as normal or the presence of osteitic changes of the bone.

Success was defined as proper cannulation and sufficient dilation (if the diameter of the dilated opening reached the diameter of the respective balloon catheter applied: from $3.5-7 \mathrm{~mm}$; www. acclarent.com) according to the surgeon's evaluation. Failure rate was defined as either inability to reach and/or dilate (i.e. dilation diameter did not reach the applied balloon catheter's diameter) the sinus ostium or passage in question, necessitating a standard FESS procedure. Failure was further subdivided into "insertion failure", "dilation failure" and "not tried". "Insertion failure" meant failed cannulation when the guide wire and/or the balloon catheter could not be deployed in the attempted sinus and thus the correct position could not be verified via transillumination and the balloon could not be advanced inside the sinus ostium. Dilation failure meant that the balloon could be deployed in the attempted sinus ostium but no dilation was possible or the dilation diameter was insufficient according to the surgeon's opinion and had to be further enlarged with standard instruments. "Not tried" meant that the intra-operative endoscopic findings -which could not be determined preoperatively- led to abstaining from BSP as in the following examples: a pre-existing accessory ostium of the maxillary sinus which cannot be connected to the natural ostium with balloons; the created ostium through BSP with a maximum diameter of $7 \mathrm{~mm}$ 
Table 1. Epidemiological data.

\begin{tabular}{|c|c|c|c|c|c|}
\hline & Male & SD & Female & SD & p-value \\
\hline N & $26(58 \%)$ & & $19(42 \%)$ & & \\
\hline Age & 44.1 & 15.9 & 43 & 13.2 & 0.802 \\
\hline CT-Score Total & 7.3 & 3.8 & 5.3 & 4.4 & 0.157 \\
\hline CT-Score Planned & 0.96 & 0.65 & 0.91 & 0.84 & 0.669 \\
\hline Osteitic Bone & $8(30.8 \%)$ & & $2(10.5 \%)$ & & 0.126 \\
\hline Previous OP & 7 (26.9\%) & & 9 (47.4\%) & & 0.118 \\
\hline
\end{tabular}

Data showing gender distribution ( $\mathrm{N}$ and \%), age (means and SD), Lund-Mackay CT-scores (means and SD of total sinuses and sinuses planned for $\mathrm{BSP}$ ), undergone previous surgeries (OP) and osteitic bone present with respective $\mathrm{p}$-values for gender differences.

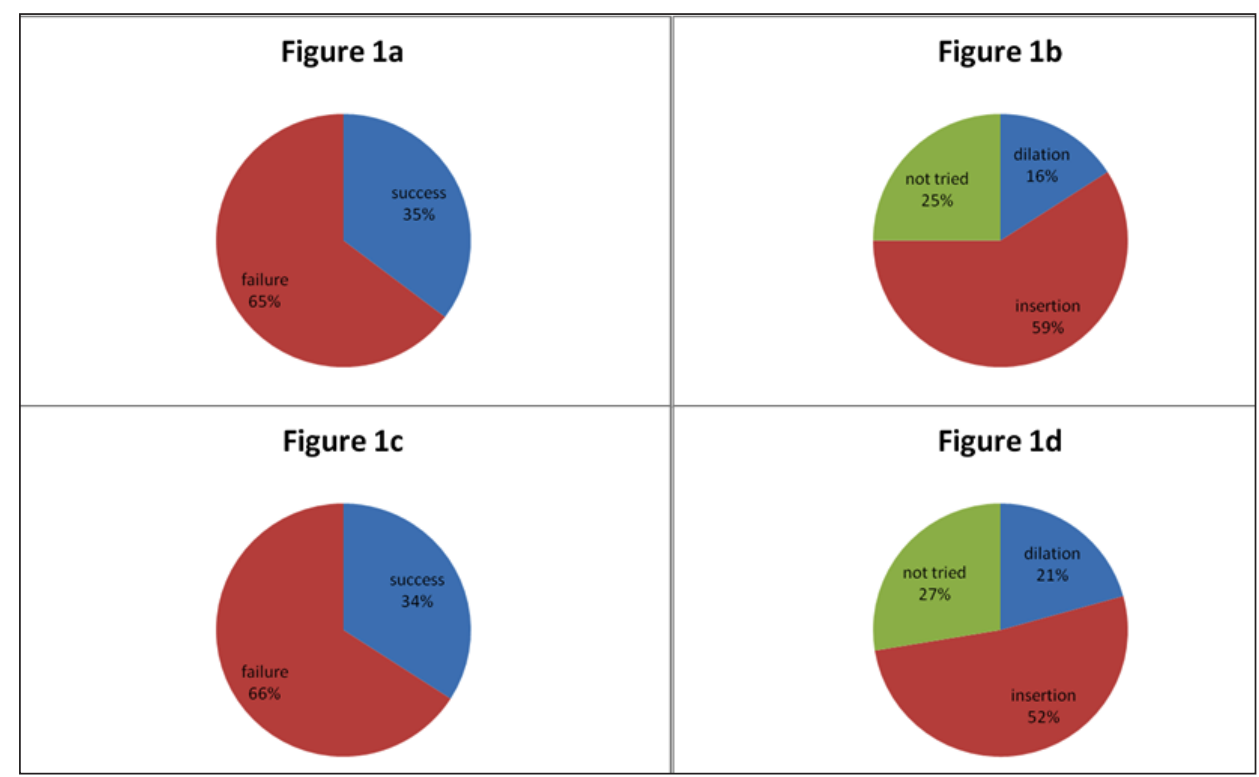

Figure 1. a) Pie chart of success and failure rates in "Balloon-Only" procedures for all sinuses. b) Detailed pie chart with failure rate in detail according to dilation, insertion and not tried failures for "Balloon-Only" procedures in all sinuses. c) Pie chart of success and failure rates in "Hybrid" procedures for all sinuses. d) Detailed pie chart with failure rate in detail according to dilation, insertion and not tried failures for "Hybrid" procedures in all sinuses.

would have been too small for necessary manipulations as in fungus balls which had not been evident on CT; massive pathology (mucosal swelling or cysts) in the middle meatus; narrow anatomic configuration of the middle or common nasal meatus which made the advancement of balloon catheters impossible.

\section{Statistical analysis}

Statistical analysis was performed with SPSS 18.0 software (SPSS Inc., Chicago, IL, USA). Values are presented as means with standard deviation. Chi-square test was used for analyzing associations of categorical values and Mann-Whitney-U test was used for analysis of differences in Lund-Mackay scores between successful and failed balloon sinuplasties. A p-value $<0.05$ was considered significant.

\section{Results}

Forty-five $(n=45)$ patients were included in this study with a gender distribution of 26 (58\%) male patients and 19 (42\%) female patients. Mean age at presentation was 43.4 years (Standard deviation (SD): 14.6 years). Mean Lund-Mackay score was 6.5 (SD: 4.1). Sixteen (35\%) patients had previously been operated on by a standard FESS procedure, 39 (65\%) were primary cases. No patient was operated via external or combined approaches (Table 1).

In 45 patients, a total of 112 sinuses were planned for BSP equaling an average of 2.5 sinuses per patient. Of the 112 sinuses, 68 (60\%) were planned as a "Balloon-Only" procedure and 44 (40\%) were planned as a "Hybrid" procedure.

After these 45 patients, the study was abandoned for reasons 
Table 2. Number of planned balloon sinuplasties.

\begin{tabular}{|c|c|c|c|c|c|c|c|c|c|c|c|}
\hline \multirow[b]{2}{*}{ Sinus } & \multirow[b]{2}{*}{ planned } & \multicolumn{2}{|c|}{ Success } & \multicolumn{2}{|c|}{ Failure } & \multicolumn{2}{|c|}{ Insertion } & \multicolumn{2}{|c|}{ Dilation } & \multicolumn{2}{|c|}{ Not tried } \\
\hline & & $\mathbf{N}$ & $\%$ & $\mathbf{N}$ & $\%$ & $\mathbf{N}$ & $\%$ & $\mathbf{N}$ & $\%$ & $\mathbf{N}$ & $\%$ \\
\hline \multicolumn{12}{|c|}{ Balloon Only } \\
\hline Total & 68 & 24 & 35 & 44 & 65 & 26 & 59 & 7 & 16 & 11 & 25 \\
\hline Frontal & 38 & 16 & 42 & 22 & 58 & 15 & 68 & 4 & 18 & 3 & 14 \\
\hline Maxillary & 19 & 2 & 10 & 17 & 90 & 7 & 41 & 2 & 12 & 8 & 47 \\
\hline Sphenoid & 11 & 6 & 55 & 5 & 45 & 4 & 80 & 1 & 20 & 0 & 0 \\
\hline \multicolumn{12}{|l|}{ Hybrid } \\
\hline Total & 44 & 15 & 34 & 29 & 66 & 15 & 52 & 6 & 21 & 8 & 27 \\
\hline Frontal & 35 & 15 & 43 & 20 & 57 & 14 & 70 & 5 & 25 & 1 & 5 \\
\hline Maxillary & 5 & 0 & 0 & 5 & 100 & 0 & 0 & 1 & 20 & 4 & 80 \\
\hline Sphenoid & 4 & 0 & 0 & 4 & 100 & 1 & 25 & 0 & 0 & 3 & 75 \\
\hline
\end{tabular}

Success and failure rates for "Balloon-Only" and "Hybrid" procedures. Failure rate is further subdivided into insertion, dilation and "not tried" failure.

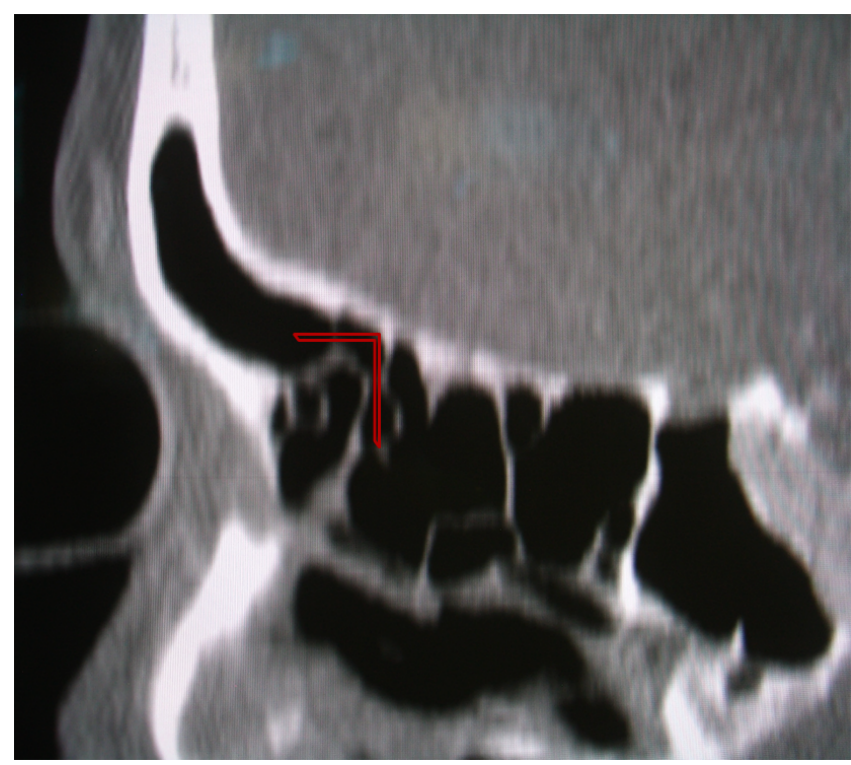

Figure 2. a) Sagittal CT-scan of a frontal recess and frontal sinus where transition-angle is $90^{\circ}$ (as indicated with red lines), Balloon insertion failed due to problems getting the guide wire around the curve when inserting the appropriate guide catheter in the middle meatus (symbolic image).

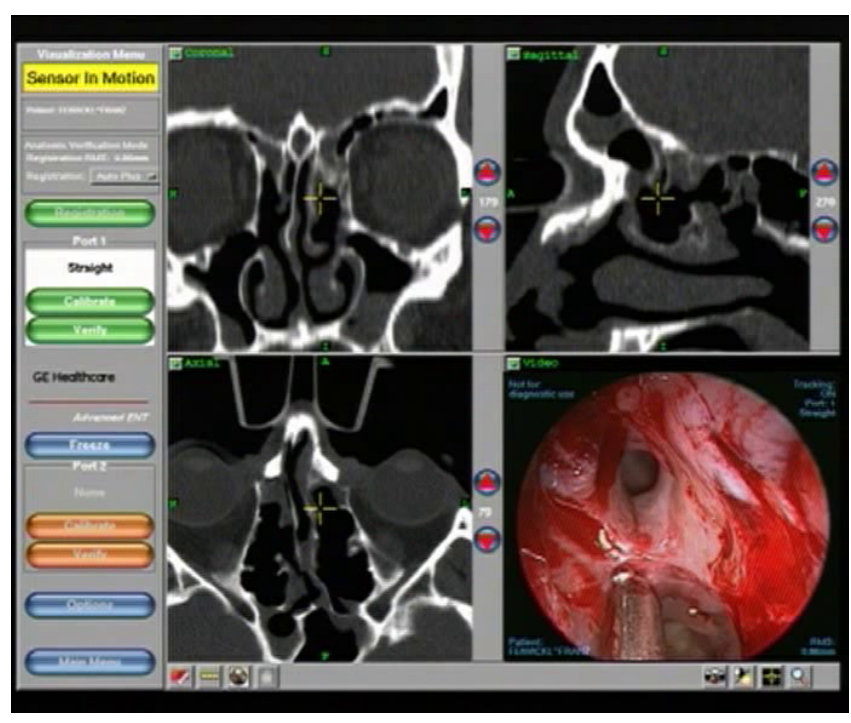

Figure 2. b) Computer assisted navigational image (InstaTrak ${ }^{\circledR} 3500$ Plus by GE Healthcare, Vienna, Austria) of an intraoperative situation where insertion of the guide wire intro frontal sinus failed due to a naso-frontal angle of $90^{\circ}$, though the case was considered ideal for BSP preoperatively. explained below, in accordance with the study protocol approved by the Medical University Ethics Committee.

\section{Balloon-Only procedures}

In total, 68 sinuses were considered feasible for the "Balloon-Only" procedure. Successful dilation and cannulation was achieved in 24 sinuses equaling a success rate of $35 \%$. In the 44 sinuses planned for the "Balloon-Only" procedure, BSP failed, equaling a failure rate of $65 \%$. In detail, insertion failed in 26 (59\%), dilation failed in 7 (16\%) and BSP was "not tried" in 11 (25\%) of all sinuses (Figures $1 \mathrm{a}$ and $\mathrm{b}$ ). For the frontal sinuses, 38 were considered "Balloon-Only" procedures with successful dilation in 16 sinuses 
Table 3. Success and failure rates for all sinuses.

\begin{tabular}{|lccccc|}
\hline Balloon only & & & & \\
& Success & SD & Failure & SD & p-value \\
\hline & $24(35 \%)$ & & $44(65 \%)$ & & \\
\hline CT-score & 0.71 & 0.7 & 0.86 & 0.7 & 0.377 \\
\hline Osteitic bone & $1(4.2 \%)$ & & $9(20.5 \%)$ & & 0.07 \\
\hline Previous surgery & $10(41.7 \%)$ & & $16(36.4 \%)$ & & 0.667 \\
\hline Hybrid & & & & & \\
\hline & & & & & \\
\hline CT-score & $15(34 \%)$ & & $29(66 \%)$ & & 0.293 \\
\hline Osteitic bone & $5(33.3 \%)$ & & $16(55.2 \%)$ & & 0.169 \\
\hline Previous surgery & $3(20 \%)$ & & $14(48.3 \%)$ & & 0.068 \\
\hline
\end{tabular}

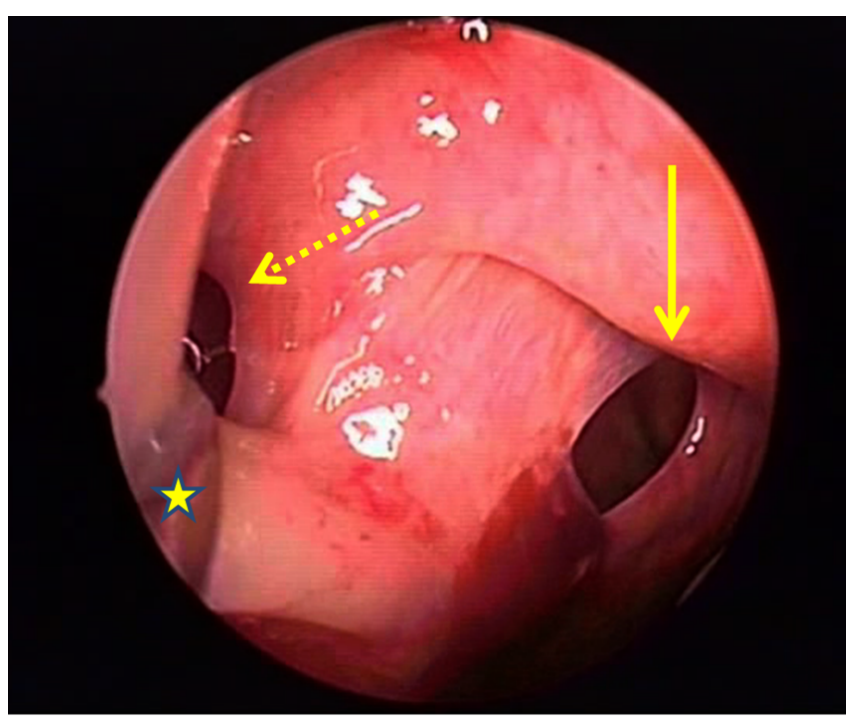

Upper part: "Balloon-Only" approach; Lower part: "Hybrid" approach.

Differences of success and failures according to the mean Lund-Mackay CT-score (of the planned sinuses), osteitic bone present and undergone previous surgery with respective $p$-values.

Figure 3. Intraoperative endoscopic view onto a maxillary sinus on the right after failed insertion with BSP by creating an accessory ostium (yellow full arrow) with pre-existing natural ostium (dotted arrow) behind the uncinate process which is deflected with a Lusk -seeker (star).

equaling a success rate of $42 \%$. In 22 frontal sinuses, BSP failed equaling a failure rate of $58 \%$. In detail, insertion failed in 15 of $22(68 \%)$, dilation failed in 4 of $22(18 \%)$ and in 3 of $22(14 \%)$ of frontal sinuses BSP was "not tried". For the maxillary sinus, 19 were planned for BSP with successful dilation in $2(10 \%)$ and failure in 17 (90\%). In detail, 7 (41\%) insertions failed, 2 (12\%) dilations failed and in 8 (47\%) BSP was "not tried". For the sphenoid sinus, 11 were planned for BSP. Successful dilation was achieved in 6 sinuses giving a success rate of 55\%. In 5 (45\%) sphenoid sinuses, BSP failed due to insertion in 4 of $5(80 \%)$ and dilation in 1 of $5(20 \%)$ sinus (Table 2).

\section{Hybrid procedures}

In total, 44 sinuses were planned for the "Hybrid" procedures. Successful dilation and cannulation was achieved in 15 sinuses equaling a success rate of $34 \%$. In 29 sinuses, BSP failed equaling $66 \%$. In detail, insertion failed in 15 of 29 (52\%), dilation failed in 6 of 29 (21\%) and BSP was "not tried" in 8 of 29 (27\%) of all failures (Figure 1c and d). For the frontal sinuses, 35 were planned for the "Hybrid" procedure with successful dilation in 15 sinuses equaling a success rate of $43 \%$. In 20 frontal sinuses BSP failed, equaling a failure rate of $57 \%$. In detail, insertion failed in 14 of $20(70 \%)$, dilation failed in 5 of $20(25 \%)$ and in 1 of 20 (5\%) of all frontal sinuses BSP was "not tried". For the maxillary sinuses, 5 were planned for BSP with successful dilation in $0(0 \%)$ and failure in 5 (100\%). In detail, 1 of 5 (20\%) dilation failed and in 4 of 5 (80\%) BSP was "not tried". For the sphenoid sinus, 4 were planned for BSP, with successful dilation in $0(0 \%)$ and failure in 4 (100\%). In detail, 1 of 4 (25\%) insertion failed and in 3 of 4 (75\%) BSP was "not tried" (Table 2).

Statistical analysis revealed no significant differences in severity of Lund-Mackay CT scores between successful and failed balloon sinuplasties (total sinuses: $p=0.068$; "Balloon-Only": $p=$ 0.377, "Hybrid": $p=0.293$ ), no association between success and failure of BSP could be seen with regard to osteitic changes of cells analysed on CT-scans for "Balloon-Only" and "Hybrid" procedures. There was no significant association in success rates between previously operated patients and primary cases (total sinuses: $p=0.6$, "Balloon-Only": $p=0.667$, "Hybrid": $p=0.068$ ) (Table 3). Success rates between "Balloon-Only" or "Hybrid" procedures were not significantly different $(p=0.943)$.

An angle around $90^{\circ}$ (or more acute) between the frontal recess (cells) and the frontal sinus could be identified as an obstacle for the guide wire reaching the frontal sinus properly (Figure 2). No intra- or postoperative adverse events were recorded.

\section{Discussion}

Since its introduction in 2002, balloon sinuplasty has been a topic of controversy among rhinologists worldwide ${ }^{(1,10)}$. On the 
one hand there were enthusiastic reports; on the other hand the technique was criticized for promises made in the treatment of chronic rhinosinusitis without strong evidence based data ${ }^{(2)}$. Initial studies on cadaveric specimen showed good feasibility and safety for balloon techniques. Bolger and Vaughan successfully dilated 31 out of 31 sinus ostia without any complication such as orbital or skull base penetration ${ }^{(12)}$. The shortcomings of cadaver studies, however, are that the surgeon is not confronted with bleeding or mucosal swelling hampering the view. Moreover, post mortem changes can make the mucosa atrophic, thus pathways are more readily visible and accessible in the anterior ethmoid. The middle turbinate's elasticity is also higher so more space is available by deflecting it with the guide catheter, facilitating the approach with this technique.

Brown and Bolger ${ }^{(7)}$ published a feasibility study on ten patients successfully dilating 18 out of 18 attempted sinuses. In this study, they successfully dilated three frontal sinuses. The frontal was the most frequently attempted and at the same time most difficult sinus in our study. In contrast to Brown and Bolger, we had planned 38 frontal sinuses and failed in 22, although our surgeons were trained and certified in balloon techniques by the manufacturer and are very experienced in endonasal procedures. Apart from the ease of cannulation, Brown and Bolger saw an advantage of BSP in avoiding circumferential trauma, reduced bleeding and removal of mucosa as in FESS, though they admitted that long-term problems from applying pressure on the mucosa with the balloon could not be predicted and had yet to be analysed. Another long term issue could be subsequent mucocoele formation through crushing and remodeling of bone as suggested by Taghi in his review ${ }^{(10)}$.

Reduced bleeding with BSP could be advantageous; however, we saw substantial mucosal trauma on several occasions due to insertion of the $110^{\circ}$ catheter for attempted maxillary sinus BSP. Although the bleeding was not severe, it did impair quality of vision for the surgeon and the attempted minimal traumatic approach was not always fulfilled. Another problem with the maxillary sinus we observed was that the maximal dilation $(7 \mathrm{~mm})$ would be too small for indications such as fungus balls, which were not evident on CT pre-operatively. Furthermore, we had insertion problems (41\%) because of a narrow middle meatus and the potential danger of out-fracturing the middle turbinate when advancing the $110^{\circ}$ catheter. Another problem was the creation of accessory ostia in the posterior fontanelle, because the guide wire did not enter the natural ostium (Figure 3). Stankiewicz et al. ${ }^{(13)}$ reported a success rate of $94.8 \%$ in 58 attempted maxillary sinuses using another sinus balloon dilation system (FinESS, Entellus Medical Inc., Maple Grove, MN, USA) which allows direct flexible endoscopic visualization of the ostium through a canine fossa puncture while advancing the balloon device. Brenner et al. ${ }^{(14)}$, however, noticed a false passage into the maxillary sinus after balloon dilation in 10 of
10 attempts in their cadaveric study via a transnasal balloon approach.

In the sphenoid, we experienced problems with transillumination for insertion of the catheter since the light of the guidewire could not be identified in cavities filled with thickened mucus or other significant disease. We considered positive transillumination mandatory for advancing the balloon catheter according to the technique's specifications for medico-legal reasons. Moreover, the lateral recesses of the sphenoid cannot be inspected or reached properly through a natural ostium enlarged to $7 \mathrm{~mm}$.

Because of its anatomical complexity, the frontal sinus appears the most promising sinus for BSP application. Heimgartner et al. (15) reported 104 attempted frontal sinuses with a success rate of $88 \%$ and failures in $12(12 \%)$ sinuses. According to their report anatomical variations such as agger nasi cells, frontoethmoidal cells, frontal bulla, etc. hamper balloon dilation in this region and pre-operative studies of CT scans are mandatory. Moreover, in their series they initially overlooked a frontal recess lymphoma, which was discovered after revision FESS since no material had been obtained during BSP for histopathological examination ${ }^{(15)}$. We also consider anatomical variations as problematic, especially if there is no terminal recess and the uncinate process is not attached to the orbit superiorly but to the middle turbinate or skull base. Furthermore, a $90^{\circ}$ or greater acute angle between frontal recess and frontal sinus was an obstacle for the guide wire not reaching "around the corner". Figure 2 illustrates one of these failures to insert the guide wire through the frontal recess passage into the frontal sinus, despite the fact that this revision case was considered a potential "Balloon-Only" procedure based on the pre-operative CT scans. Neither do the bone segments appear osteitic or otherwise thickened, nor would the endoscopic view be obscured (right lower quadrant of picture, taken during the image-guided procedure). The only common determinant in these cases was a naso - frontal angle of $90^{\circ}$ or less (Figure 2). Bone thickness of cells analysed on CT scans, however, was no predictor for success or failure of BSP. Catalano et al. ${ }^{(16)}$ and Luong et al. ${ }^{(17)}$ reported 29 and 6 successful frontal dilations, respectively, though Catalano excluded sinuses in which insertion was not possible and Luong's patients had all undergone at least one previous frontal sinusotomy where access to the frontal recess was created with standard instruments and not with a balloon device, thus biasing the results.

Hopkins et al. ${ }^{(18)}$ reported successful dilation in $98 \%$ of 67 sinuses in 27 patients, emphasizing in particular that they had not encountered feasibility problems. The largest prospective study to date is the CLEAR study (The Clinical Evaluation to Confirm Safety and Efficacy of Sinuplasty in the Paranasal Sinuses) with follow-up intervals of 24 weeks, one year and two years respectively ${ }^{(4,5,19)}$. After two years, 195 dilated sinuses in 65 patients 
(initially 115 patients) showed significantly improved SNOT-20 scores and Lund-Mackay scores. Initially 347 of 358 (96.9\%) sinuses were successfully dilated. Batra ${ }^{(20)}$ states in his review that the "Hybrid" group in the CLEAR study showed better SNOT20 outcomes after two years compared with the "Balloon-Only" group (0.64 vs 1.09) and they had started with a higher preoperative SNOT-20 (2.42 vs. 2.14) showing that patients additionally benefitted from the surgical part in "Hybrid" procedures. Moreover, it is important to directly compare success of BSP to standard FESS procedures as was done by Koskinen et al. ${ }^{\left({ }^{9}\right)}$ who reported slightly better outcomes for FESS in acute exacerbations and in patients with occupational or CRS-related risk factors, as well as in patients with disease(s) other than those known to be CRS-related comorbidities. Reported complication rates of BSP are negligible although rarely severe complications have been reported such as CSF-leaks ${ }^{(21)}$. In the present study no intra- or post-operative complications occurred.

In combination with FESS, balloon sinuplasty can be a useful adjunct in selected patients for chronic rhinosinusitis or patients in Critical Care with acute rhinosinusitis ${ }^{(18)}$ or patients suffering from barosinusitis ${ }^{(22)}$, and it may have a role under local anaesthesia in an office setting. However, these indications as well as the long-term results need to be further determined.

\section{Concluding remarks}

There is a striking discrepancy between some enthusiastic reports in literature and our poor success rate. A potential explanation for this could be that our institution, contrary to other studies, is both a primary and a tertiary medical care centre and many of our patients had been referred to us with a clear indication for surgery, without knowledge of which approach (BSP or FESS) was eventually performed in these patients. Thus, we can conclude that there was no bias due to the referring system. Despite our own thorough pre-operative examination and choice of the appropriate approach for each sinus by our surgeons, we were obviously unable to determine which case ultimately was suitable for balloon techniques, be it "BalloonOnly" or "Hybrid" procedures, or where standard FESS was the more appropriate technique reflected by our high balloon techniques' failure rate. This was usually due to unpredictable intra-operative findings and would place a surgeon trained only with balloon based techniques in a difficult position in a significant number of cases. This emphasizes the need for continued training in a wide range of sinonasal techniques and procedures.

Another question raised by these results concerns our surgical team's ability to perform the balloon technique properly. However, the two senior surgeons were well trained, instructed and certified by the company and a pre-study trial including 37 patients had been performed so as not to bias the present study by a learning curve. However, it is worth noting that the success rates in the pre-study were almost identical to the ones of the present study (Data presented by H. Stammberger at the 13th British Academic Conference in Otolaryngology, 2009).

It could be argued that had we completed the study in 200 patients, the results for BSP may have improved, but we did not feel it was ethical to continue in the light of the high failure rate. Our actual patient cohort could also be unrepresentative of those patients reported in the literature, perhaps in the criteria applied for surgical intervention. However, our patients were drawn from a population of about 1.3 million inhabitants and fulfilled the criteria for diagnosis and had failed medical therapy in CRSsNP which would seem to be the appropriate target group for these techniques. Thus, based on this present study, we cannot recommend routine BSP as a replacement of standard FESS for frontal, maxillary and sphenoid sinuses in CRSsNP, though perhaps this was never the intention of the technique and it should be regarded rather as simply another part of our surgical armamentarium.

Naturally, these questions cannot be answered by the present study but further multicentre prospective trials are urgently needed with strictly agreed inclusion and exclusion criteria to determine the role of the BSP techniques and technology.

\section{Conclusion}

Based on the results of the Graz study, Balloon sinuplasty cannot be recommended as a replacement for standard FESS procedures, but may be used as an adjunct. In our setting, a failure rate of $65 \%$ for "Balloon-Only" and of $66 \%$ for "Hybrid" procedures in patients with well documented CRS occurred which was not acceptable for our study group. The study therefore, initially scheduled for 200 consecutive patients, was abandoned after 45 patients.

\section{Acknowledgement}

Our study was not sponsored, however, equipment was provided free of charge by the Acclarent company.

\section{Authorship contribution}

PVT: Study design, data acquisition, writing, statistical analysis HS: Study design, data acquisition, writing $\mathrm{HB}, \mathrm{WH}, \mathrm{CS}$, WK: Data acquisition GPH: Statistical analysis

\section{Conflict of interest}

The authors state that there is no conflict of interest regarding this manuscript. 


\section{References}

1. Siow JK, Al Kadah B, Werner JA. Balloon sinuplasty: A current hot topic in rhinology. Eur Arch Otorhinolaryngol. 2008; 265: 509-511.

2. Lanza DC, Kennedy DW. Balloon sinuplasty: Not ready for prime time. Ann Otol Rhinol Laryngol. 2006; 115: 789-792.

3. Levine H, Rabago D. Balloon sinuplasty: A minimally invasive option for patients with chronic rhinosinusitis. Postgrad Med. 2011 123: 112-118.

4. Bolger WE, Brown CL, Church CA, et al. Safety and outcomes of balloon catheter sinusotomy: A multicenter 24-week analysis in 115 patients. Otolaryngol Head Neck Surg. 2007; 137: 10-20.

5. 5. Weiss RL, Church CA, Kuhn FA, Levine HL, Sillers MJ, Vaughan WC. Long-term outcome analysis of balloon catheter sinusotomy: Two-year follow-up. Otolaryngol Head Neck Surg. 2008; 139: S38-46.

6. Fokkens W, Lund V, Mullol J, Bachert C et a European Position Paper on Rhinosinusitis and Nasal Polyps group. European position paper on rhinosinusitis and nasal polyps 2012. Rhinol Suppl. 23; 2012; 1-298.

7. Brown $\mathrm{CL}$, Bolger WE. Safety and feasibility of balloon catheter dilation of paranasal sinus ostia: A preliminary investigation. Ann Otol Rhinol Laryngol. 2006; 115: 293-301.

8. Friedman M, Schalch P, Lin HC, Mazloom $\mathrm{N}$, Neidich $\mathrm{M}$, Joseph NJ. Functional endoscopic dilatation of the sinuses: Patient satisfaction, postoperative pain, and cost. Am J Rhinol. 2008; 22: 204-209.

9. Koskinen A, Penttila M, Myller J, et al. Endoscopic sinus surgery might reduce exacerbations and symptoms more than balloon sinuplasty. Am J Rhinol Allergy. 2012; 26: 150-156.

10. Taghi AS, Khalil SS, Mace AD, Saleh HA Balloon sinuplasty: Balloon-catheter dilation of paranasal sinus ostia for chronic rhinosinusitis. Expert Rev Med Devices. 2009; 6 377-382.

11. Lund VJ, Kennedy DW. Staging for rhinosinusitis. Otolaryngol Head Neck Surg. 1997; 117: S35-40.

12. Bolger WE, Vaughan WC. Catheter-based dilation of the sinus ostia: Initial safety and feasibility analysis in a cadaver model. Am J Rhinol. 2006; 20: 290-294.

13. Stankiewicz J, Tami T, Truitt T, Atkins J, Liepert D, Winegar B. Transantral, endoscopically guided balloon dilatation of the ostiomeatal complex for chronic rhinosinusitis under local anesthesia. Am J Rhinol Allergy. 2009; 23: 321-327.

14. Brenner PS, Abadie WM, Weitzel EK, Thomas RF, McMains KC. Unexpected consequences of transnasal balloon dilation of the maxillary ostium. Int Forum Allergy Rhinol. 2011; 1: 466-470.

15. Heimgartner S, Eckardt J, Simmen D, Briner HR, Leunig A, Caversaccio MD. Limitations of balloon sinuplasty in frontal sinus surgery. Eur Arch Otorhinolaryngol. 2011

16. 16. Catalano PJ, Payne SC. Balloon dilation of the frontal recess in patients with chronic frontal sinusitis and advanced sinus disease: An initial report. Ann Otol Rhinol Laryngol. 2009; 118: 107-112.

17. Luong A, Batra PS, Fakhri S, Citardi MJ Balloon catheter dilatation for frontal sinus ostium stenosis in the office setting. Am
Rhinol. 2008; 22: 621-624.

18. Hopkins C, Noon E, Bray D, Roberts D. Balloon sinuplasty: Our first year. J Laryngol Otol. 2011; 125: 43-52.

19. Kuhn FA, Church CA, Goldberg AN, et al. Balloon catheter sinusotomy: One-year follow-up--outcomes and role in functional endoscopic sinus surgery. Otolaryngol Head Neck Surg. 2008; 139: S27-37.

20. Batra PS. Evidence-based practice: Balloon catheter dilation in rhinology. Otolaryngol Clin North Am. 2012; 45: 993-1004.

21. Tomazic PV, Stammberger H, Koele W Gerstenberger C. Ethmoid roof CSF-leak following frontal sinus balloon sinuplasty. Rhinology. 2010; 48: 247-250.

22. Brehmer D. Catheter-based balloon dilatation of the frontal, maxillary, and sphenoid ostia: A new procedure in sinus surgery. HNO. 2008; 56: 65-70.

Peter Valentin Tomazic, MD

ENT-University Hospital Graz

Medical University Graz

Auenbruggerplatz 26

$8036 \mathrm{Graz}$

Austria

Tel: +43-316-385-3448

Fax: $+43-316-385-3425$

E-mail:peter.tomazic@medunigraz.at 